\title{
A High Dynamic Performance PMSM Sensorless Algorithm Based on Rotor Position Tracking Observer
}

\author{
Tianmiao Wang ${ }^{1, a}$,Shiqian Dong ${ }^{2, b}$, Yong Tao ${ }^{1, c^{*}}$, Ye Wang ${ }^{2, d}$ and Zhongyuan \\ Chen $^{2, e}$ \\ ${ }^{1}$ School of Mechanical Engineering and Automation, Beihang University, Beijing, P. R. China \\ ${ }^{2}$ Ninebot Inc., Beijing, P. R. China \\ aitm@buaa.edu.cn, bdongshiqian88@sina.com, 'taoy@buaa.edu.cn, ${ }^{\mathrm{d}}$ Cid.wang@ninebot.com, \\ ezy.chen@ninebot.com
}

\begin{abstract}
Keywords: Permanent magent synchronous motor, Rotor position estimate, Motor driver system, Manned self-balancing vehicle

Abstract. In this paper a new permanent magent synchronous motor (PMSM) rotor position estimate algorithm is discussed. The algorithm adjusts the estimated rotor position PI controller, makes the rotor back electromotive force produced by rotor permanent magnet on $\mathrm{d}$ axis veins. Compared with other PMSM position estimate algorithms, the algorithm has a higher dynamic performance and realizes stable rotor position / speed estimate even under high speed and load variations. The implementation of this algorithm is very simple, it does not change the original control algorithm structure of motor driver, and only consumes very little computational resources and is very suitable for low-cost PMSM driver system whose resources are limited. And the algorithm is robust to parameter variation. The algorithm has been test in the motor driver system of manned self-balancing vehicle.
\end{abstract}

\section{Introduction}

In the electric vehicles, permanent magent synchronous motor is widely applied due to its advantages, such as high power density, long life, low noise and easy to control, etc. In order to achieve smooth and high efficient operation effects, the driver system uses field oriented control (FOC) algorithm, and FOC control needs to get the rotor position by position sensor. The position sensor generally used is Hall sensor, and the Hall sensor has some probability of failure. Once one or several Hall sensors of the PMSM fails, the rotation of the rotor cannot be obtained correctly, which easily leads to serious consequences: if not severe, the excessive current will damage the driver, if severe, the motor will be suddenly stuck and cause the accident. In order to avoid above situation, rotor position sensor estimate algorithm of various dispense rotor position have been developed.

Rotor position estimate algorithm can be divided into estimate algorithm based on PMSM mathematical model and high-frequency injection method, in which the high-frequency injection method is not suitable for using low-cost driven civilian electric vehicles due to the larger noise and requirement of high-precision current sampling hardware. However, the model-based algorithm estimates rotor position based on back electromotive force, and most of them are applied to electric vehicles working at high speed. There are a lot of researches on the rotor position estimate method model-based algorithm. The algorithm widely applied in the engineering is Luenberger state observer algorithm [3]. This algorithm uses the linear state observer to extract stator BEMF on $\alpha$ and $\beta$ axis and extract rotor position and rotation rate information from BEMF signal of 90 degrees between phase-locked loop and phase position. However, because the observer and phase-locked loop bandwidth is relatively low, the dynamic performance of this method is very limited. It will generate larger estimate error when speed load mutates. In order to improve the response speed and robustness of Lunberger observer method, there is research which improves the observer into sliding mode observer (SMO) [4]. The output of sliding mode observer contains high-frequency vibration of sliding mode switching and runs not smoothly enough. In order to eliminate vibration by introducing low-pass filter, it will reduce the bandwidth of the system and affect the dynamic performance. Another approach which has been extensively studied is extended Kalman filter method. The 
establishment of this method includes complete mathematical model of motor mechanical and electrical characteristics. When the actual working condition of motor is consistent with the model used by EKF, it will have better dynamic performance. However, EKF generally regards the mechanical part of the motor as moment of inertia and mechanical load unchanged; this is usually quite different from the actual situation. The difference between theory and practice will greatly reduce estimate accuracy. Besides, EKF requires larger calculation amount and of cannot run at a high enough speed in microcontroller of low-cost PMSM driver, so it is not commonly used in the project. Joohn-Sheok Kim [5] proposes the algorithm based on "current steady state". Based on the error between estimate rotor position and the actual rotor position, the algorithm establishes establish the motor mathematical model and introduces the concept of "electrical steady-state", that is, the running frequency of estimator is lower than the control frequency of the motor current close loop, so that the current in stator has stabilized and converged to the preset current value when conducting the rotor position estimate, thereby eliminating the differential items in current in model, which greatly simplifies the algorithm extracting BEMF. The algorithm is with good dynamic performance, but it is also limited in some practice. The algorithm requires the controller of current loop to have short adjustment time; therefore, the current loop sampling and control frequency should be high enough. However, in the low-cost PMSM driver, due to the performance limitation of the microcontroller and inverter, the current sampling and control frequency are not too high, and therefore, it is difficult to achieve the requirement of "electrical steady-state"; Moreover, some drivers do not have current close loops, but directly give cross-axis voltage driven motor to rotate, this algorithm cannot be used.

Jul-Ki Seok[1] proposes that regard voltage output by d-axis current controller in driver as the error of estimate angle and make the angle error be zero by tracking observer estimate rotor position[1]. The algorithm has the ideal dynamic performance and robustness and its structure is simple, but there still are the following shortcomings: Firstly, the control algorithm still requires the driver to have current regulator, and therefore, it cannot be directly applied in the driver which have no current loop, the application range subject to certain restrictions; Secondly, in this paper, d-axis current is controlled by feedback regulator and feedforward compensation, the rotor position estimator uses the output voltage of feedback controller as estimate error signal, but the author in this paper does not indicate how the feedforward controller should be designed to make the output of feedback controller correctly reflect estimate error; Thirdly, this algorithm can work only when the rotor is at certain rotational speed. When the motor is started at zero speed, it must obtain the initial position by position sensor or high frequency injection method. After reaching a certain speed, it will switch to senseless algorithm, and the author in this paper does not give the strategy of switching driving mode.

In this paper, the algorithm uses a set of "estimation coordinate system" for independent of the coordinate system used by driving algorithm to conduct angular estimate, and therefore, it is separated from the driver's current regulator. No matter what the driving algorithm is adopted, only obtaining stator drive voltage and line current can estimate the rotor position, therefore, it has a strong portability. In this paper, the author analyzes the compensation amount of d-axis voltage and explains the physical meaning of such compensation. The author in this paper also analyzes the switching from startup mode to senseless operating mode and designs hysteresis switching strategy to eliminate vibration which may be aroused in switching.

\section{PMSM Mathematical Model}

Coordinate system based on stator positon, actual rotor position and estimation rotor position are defined as Fig. 1. 


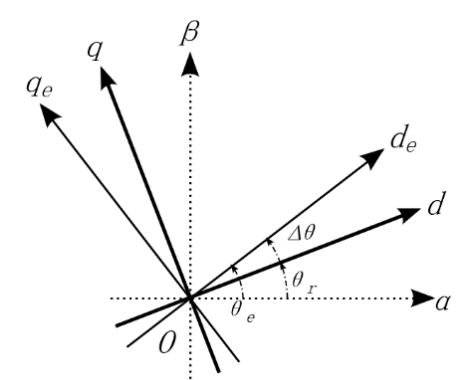

Fig. 1 the coordinate system based on stator positon, actual rotor position and estimation rotor position PMSM mathematical model based on actual rotor positon is:

$$
\left[\begin{array}{l}
u_{d} \\
u_{q}
\end{array}\right]=\left[\begin{array}{ll}
R & p L_{d}-\omega_{r} L_{q} \\
R & p L_{q}+\omega_{r} L_{d}
\end{array}\right]\left[\begin{array}{l}
i_{d} \\
i_{q}
\end{array}\right]+\left[\begin{array}{c}
0 \\
\omega_{r}
\end{array}\right] \lambda_{p M}
$$

The operator notation " $p$ " means the differential operator with respect to time; $\lambda_{p a}$ is the flux generated by permanent magents; $L_{\mathrm{s}}, L_{\bar{z}}$ and $R$ are the inductance and resistance, respectively along direct quadrature axis.

Since the electrical steady state is assumed, current derivateives can be ignored. The error between real rotor position and estimate rotor is defined as $\Delta \theta=\theta_{z}-\theta_{s}^{*}$, which $\theta_{r}$ is real rotor position and $\theta_{i}$ is estimate rotor position. To derive the relationship of voltage and current between real and estimation coordinate system, a rotation matrix is multiplied to equation (1)[2]:

$$
\begin{aligned}
& {\left[\begin{array}{l}
u_{d e} \\
u_{q u}
\end{array}\right]=\left[\begin{array}{cc}
R-\omega_{q} \sin \Delta \theta \cos \Delta \theta\left(L_{d}-L_{q}\right) & -\omega_{\theta}\left(L_{q} \cos ^{2} \Delta \theta+L_{d} \sin ^{2} \Delta \theta\right) \\
\omega_{\theta}\left(L_{q} \sin ^{2} \Delta \theta+L_{d} \cos ^{2} \Delta \theta\right) & R+\omega_{e} \sin \Delta \theta \cos \Delta \theta\left(L_{d}-L_{q}\right)
\end{array}\right] \cdot\left[\begin{array}{l}
i_{d \theta} \\
i_{q \theta}
\end{array}\right]+} \\
& {\left[\begin{array}{c}
-\omega_{r} \sin (\Delta \theta) \\
\omega_{r} \cos (\Delta \theta)
\end{array}\right] \lambda_{p M}}
\end{aligned}
$$

In a surface mount PMSM, there is $\Sigma_{i} \approx \Sigma_{i}=\Sigma_{i}$, so equation (2) can be simplified as:

$$
\left[\begin{array}{l}
u_{d \theta} \\
u_{q \theta}
\end{array}\right]=\left[\begin{array}{cc}
R & -\omega_{\theta} L_{s} \\
\omega_{\theta} L_{s} & R
\end{array}\right]\left[\begin{array}{l}
i_{d \theta} \\
i_{q \theta}
\end{array}\right]+\left[\begin{array}{c}
-\omega_{r} \sin (\Delta \theta) \\
\omega_{r} \cos (\Delta \theta)
\end{array}\right] \lambda_{p M}
$$

In equation(3), a relationship between voltage of direct and quadrature axis and estimation error is built. Then we can use following equation to extract estimation error:

$$
\Delta \theta \approx \sin (\Delta \theta)=-\left(u_{d \theta}-R i_{d \theta}+\omega_{\theta} L_{s} i_{d \theta}\right) / \omega_{g} \lambda_{P M}
$$

\section{The Design of Rotor Position Tracking Observer}

\section{The structure of tracking observer.}

The design purpose of sensorless estimator is to obtain the stator voltage and current, extract error between estimate position and real position, and then adjust estimation angle to make the estimation error veins.

Estimation error can be extract from equation (4). But at extremely low speed, the denominator of (4) is close to zero, and noise in the control loop will be amplified. To avoid this, the equation (4) can be changed to:

$$
\begin{gathered}
\Delta \theta \approx-\left(u_{d e}-R i_{d e}+\omega_{z} L_{z} i_{g \theta}\right) /\left(K \lambda_{p N}\right) \\
K=\left\{\begin{array}{cc}
\omega_{\theta}, & \omega_{\theta} \geq \omega_{0} \\
\omega_{0}, & \omega_{e}<\omega_{0}
\end{array}\right.
\end{gathered}
$$

$\omega_{0}$ is the threshold speed. If $\omega_{e}$ is lager than $\omega_{0}$, use equation(4) to extract estimation error. If $\omega_{e}$ is smaller than $\omega_{0}$, the denominator of equation(5) is pinned to $\omega_{0} \lambda_{P M}$. In this situation, estimation error is decreased to $\Delta \theta \omega_{g} / \omega_{0}$. It is obvious that if rotor speed is zero, the estimation error will always be zero, so rotor position can not be estimated. 
The structure of tracking observer is shown in Fig. 2. Fig. 2(a) shows a uint power factor field oriented controller, which current of direct axis is controlled to be zero. Fig. 2(b) shows the rotor position estimator based on tracking observer. The estimator use a "estimation coordinate system", which is independent form coordinate system based on real rotor position. Park transform is used to transform voltage and current in stator coordinate system to estimation coordinate system". Then equation (5) and (6) are used to extract estimation error. A proportional-integral regulator is introduced to make estimation error veins. After PI controller, a pole is mandatory to add to obtain a second type system, ensuring the tracking error of a ramp signal veins.

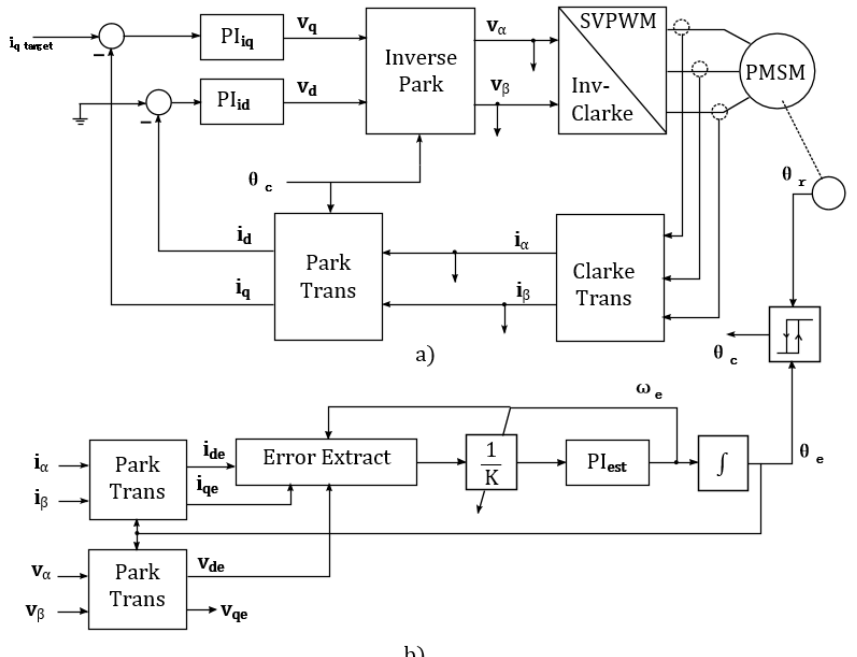

b)

Fig. 2. Sensorless PMSM driver block diagram

Because the algorithm could not maintain estimation precision at extremely low speed, and some application needs the PMSM to work in both zero speed and high speed, the rotor position sensor in the low speed operation is adopted. A switching law with hysteresis band is adopted for the switching of FOC with position sensor at very low speed and sensorless control at higher speed.

The dynamic performance of tracking observer

The open loop transfer function is:

$$
G(s)=\frac{k_{y^{s}+K_{i}}}{s^{2}}
$$

the bandwidth and phase margin can be calculated:

$$
\begin{gathered}
\left|G\left(j \omega_{g}\right)\right|=1 \\
\varphi_{m}=\arg \left[G\left(j \omega_{g}\right)\right]+\pi
\end{gathered}
$$

we can derive from $(7)(8)(9)$ that:

$$
\begin{gathered}
\omega_{g}=\sqrt{\frac{K_{p}^{n}+\sqrt{K_{p}^{4}+4 K_{i}^{2}}}{2}} \\
\varphi_{m}=\arctan \frac{K_{p}}{K_{i}} \sqrt{\frac{K_{p}^{2}+\sqrt{K_{p}^{4}+4 K_{i}^{2}}}{2}} \\
K_{p}=\omega_{g} \sqrt{\frac{\left(\tan \varphi_{m}\right)^{2}}{1+\left(\tan \varphi_{m}\right)^{2}}} \\
K_{i}=\omega_{g}^{2} \sqrt{\frac{1}{1+\left(\tan \varphi_{m}\right)^{2}}}
\end{gathered}
$$


Equation (12) (13) shows the relationship between the gain of PI controller and system dynamic performance. Higher PI gain leads to higher bandwidth and response speed, but also amplifies sampling noise; Lower PI gain makes estimation smoother but also has lower bandwidth.।

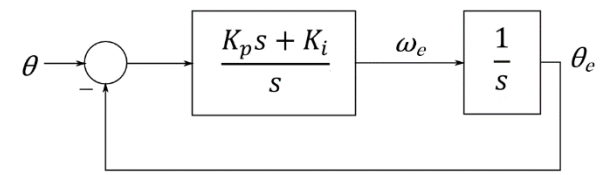

Fig. 3. Block diagram of the sensorless control scheme

\section{Simulation result}

The algorithm is tested in Matlab. To compare the performance of different rotor position estimate algorithm, a Lunberger observer method from the Freescale Semiconductor's official PMSM driver algorithm[3] is also adopted and simulated. The PMSM's electrical parameters are defined as follow:

$$
L_{Q}=L_{G}=18.94 \mathrm{mH}_{j} \mathrm{R}=2.0 \Omega_{j} \lambda_{P M}=0.084 \text { Guass }
$$

Fig. 4 and Fig. 5 shows the speed response and estimation error when motor tracking a ramp speed command. The tracking observer method has shorter response time and smaller estimation error.

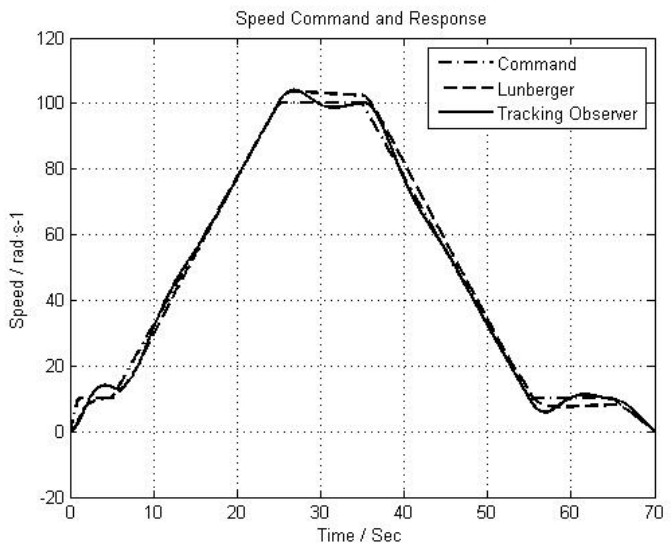

Fig. 4 Speed tracking of sensorless algorithm

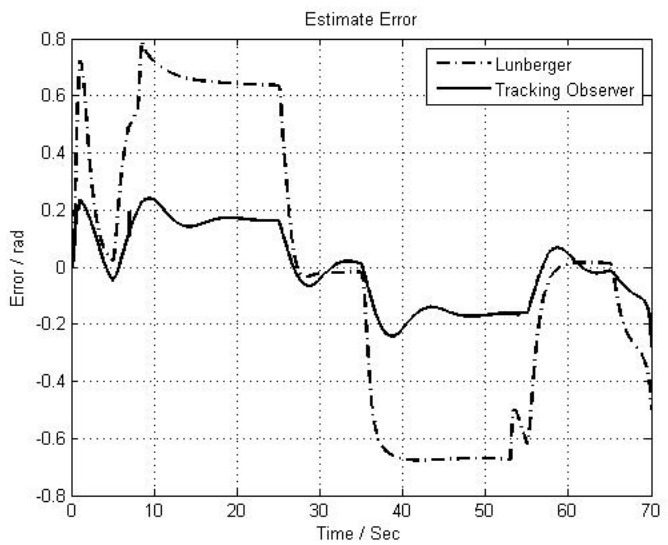

Fig. 5 Estimation error of sensorless algorithm

Fig. 6 and Fig. 7 shows the speed response and estimation error when a step load disturbance of $0.5 \mathrm{Nm}$ is added at $40 \mathrm{~s}$. The tracking observer method has shorter response time and smaller estimation error.

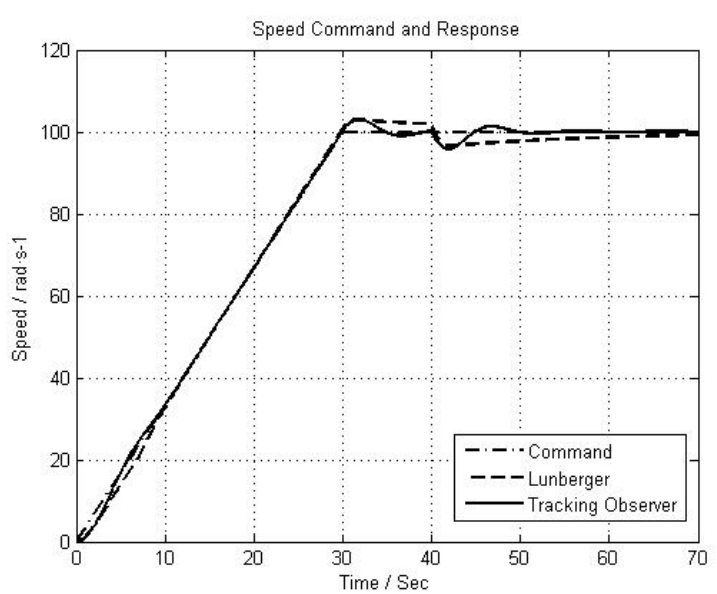

Fig. 6 Speed response under load disturbance

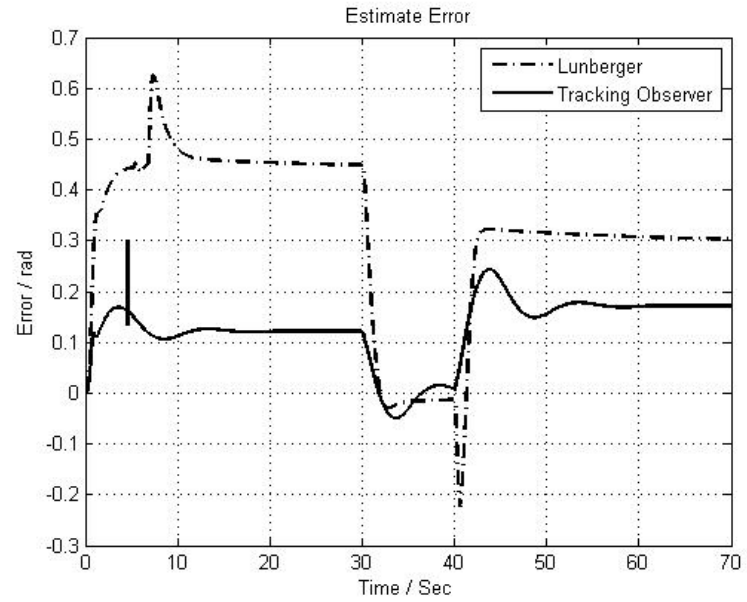

Fig. 7 Estimation error under load disturbance

The reason of Luenberger observer has larger steady state error is the algorithm assumes the rotor flux linkage is align to $\mathrm{d}$ axis, but when current of q axis increase, flux linkage will no longer align to 
$\mathrm{d}$ axis, so the estimation error increase. But the tracking observer algorithm considers the whole mathematical model of PMSM, so it has higher dynamic performance.

\section{Sensitivity to parameter variations}

\section{Variation of stator resistance.}

Consider equation (5):

$$
\Delta \theta \approx-\left(u_{d \theta}-R i_{d \theta}+\omega_{\theta} L_{s} i_{q \theta}\right) /\left(K \lambda_{P M}\right)
$$

For a UPF-FOC, current of $\mathrm{d}$ axis is zero, so the $R i_{d \theta}$ term is zero at steady state, and has no effect to rotor position estimation.

\section{Variation of Inductance}

Consider a variance of $\mathrm{L}$ :

$$
\begin{gathered}
L^{\prime}=L+\Delta L \\
\Delta \theta^{\prime} \approx-\left(u_{d \theta}-R i_{d \theta}+\omega_{\theta} L_{g}{ }^{{ }_{i} i_{\theta}}\right) /\left(K \lambda_{P M}\right)
\end{gathered}
$$

We can derive that:

$$
\Delta \theta^{\prime} \approx \Delta \theta+\frac{\Delta L i_{Q P}}{\lambda_{P M}}
$$

Equation(16) shows that estimation error will become larger under heavy load.

\section{Variation of permanent magnet}

When the estimator reaches steady state, there is $u_{d \theta}=R i_{d \theta}-\omega_{g} L_{g} i_{d \theta}$, so the variance of $\lambda_{p M}$ has no effect to rotor position estimation.

\section{Conclusions}

In this paper a rotor position tracking observer is discussed. The algorithm adjusts the estimated rotor position PI controller, makes the rotor back electromotive force produced by rotor permanent magnet on $\mathrm{d}$ axis veins. Compared with other PMSM position estimate algorithms, the algorithm has a higher dynamic performance and realizes stable rotor position/speed estimate even under high speed and load variations.

\section{References}

[1] Lee J K, Seok J K, Lee D C. Sensorless Speed Control Of Nonsalient Permanent-Magnet Synchronous Motor Using Rotor-Position-Tracking Pi Controller[J]. IEEE Transactions on Industrial Electronics, 2006, 5(2):4024 - 4029.

[2] Matti Eskola. Speed and Position Sensoless Control of Permanent Magnent Synchronus Motors in Matrix Converter and Voltage Source Converter Applications. Tampere University of Technology, 2006, 77-82.

[3] Freescale Semiconductor. DRM148-Sensorless PMSM Field-Oriented Control - Design Reference Manual. Freescale Semiconductor, 2014.

[4] Zhao Y, Qiao W, Wu L. Position extraction from a discrete sliding-mode observer for sensorless control of IPMSMs[C]// 2012 IEEE International Symposium on Industrial Electronics (ISIE), 2012:725-730.

[5] Kim J S, Sul S K. New Approach for High Performance Pmsm Drives Without Rotational Position Sensors[C]// APEC '95. Conference Proceedings of Applied Power Electronics Conference and Exposition, 1995:381-386. 